\title{
Comprehensiveness of UML in Reservoir Automation System Using Zigbee and GSM
}

\author{
${ }^{1}$ Umasankar Ch., ${ }^{2}$ Sateesh Kumar Reddy Ch. ${ }^{3}$ G. Anand Babu \\ Dept. of Electronics \& Communication Engineering, Universal College of Engineering \& Technology, Guntur, \\ AP, India
}

\begin{abstract}
Rapid development of automation and measuring techniques have left no clues what so ever for automatic recording of the data in the meter reading instruments in every field. One field where automatic measuring hasn't developed as much as in other fields is in measuring the water flow and water level in water reservoirs. Utilities also hope that the development of new technologies to solve the problems they encountered in the practical work about measuring and no reliable protection of accuracy and real time; and enable both user-friendly and improving public sector efficiency and management level. This Paper proposes a novel method for measuring the water level in the reservoirs which collects the data from different Zigbee nodes at the project site and through a GSM Module it is transported to the PLC, which enables us to monitor and control the water level in the reservoir. As GSM modules are used there is feasibility for a single server to maintain many reservoirs in a stipulated area.
\end{abstract}

Keywords: Reservoir automation system (RAS), Automation system, Zigbee, GSM, Unified Modeling Language (UML) and PLC's.

\section{Introduction}

In recent years, Model-Driven Architecture (MDA) has been become a populate software system development approach. The MDA approach in software engineering is more important and has been provided a good support and consolidation for the automatic software development, the MDA separates business and application logic from underlying platform. The platform independent model (PIM) is transformed into the platform specific model (PSM) using certain transformation rules and PSM is transformed into application code with code generators. The aim of Model driven Architecture is to develop application automatically. To this aim, the software development phase needs a modeling approach to provide completed models for model transformation purpose. The developers' community of software for embedded systems tends to use graphical methods for modeling and program specification such as the Unified Modeling language, since UML is a suggestive graphical notation covering the most relevant system and engineering aspects, and since it has become an industrial standard of object oriented modeling.

In UML, the paradigms of modern software development are integrated into a comprehensive and widely accepted visual language, providing a complete methodology to design systems ranging from small controllers to large and complex open systems. With the ever increasing complexity of embedded control systems, design and implementation have to fulfill demanding requirements with respect to functionality, timing, reliability, cost, safety and security. To meet these requirements, an integrated development environment based on an extended UML is designed. It aims at providing a comprehensive set of methods and tools for all development phases of complex embedded control systems. For the environment to be fully integrated, the gaps between the applicability of UML models for proper modeling as well as for validation, verification, simulation and code generation need to be filled. To enable domain-specific specification, in form of profiles UML is enhanced by comprehensive sets of constructs addressing the aspects real time, distribution and safety characteristic for embedded control systems. Corresponding models should automatically be translated to several other model notations.

Here, a UML profile for simulation models is defined, which is process-oriented and based on extensions of UML oriented at PEARL for distributed systems and real-time extensions of UML state charts which enable to simulate real-time constraints. This UML profile is expected to automatically translate UML models into simulation models described as XML documents, utilizing existing model analysis tools for quantitative system analysis without the need for individual complex and expensive formal modeling.

Wireless sensor networks are the combinative production of computer technology, communication technology and network technology. The micro-sensors can real time monitor, apperceive and collect the environmental information. In this paper, we present a remote wireless system based on wireless sensor networks and embedded technology which can solve the problems in existing Reservoir monitoring system. The system consists of wireless sensor nodes, data collector, management centre and wireless communication 
networks. By using this system, the reservoir monitoring and controlling task can be finished by the single PLC from the control room. The proposed system have abroad application foreground in the real application field.

Zigbee reservoir monitoring and controlling refers to the use of short range wireless communication technology and computer network technologies to read and process metering data automatically. Wireless reservoir monitoring and controlling technology can not only save human resources, but more importantly may improve the accuracy and enabling management sector to access to data messages timely and accurately. No cable wiring can save human and material resources, so investment is considerably economical. Wireless communication links can be quickly built, engineering cycle significantly shortened, and has better scalability compared to a wire-line system $[2,3]$.

If a fault occurs, only check wireless data module for causes quickly, and then restore the system back to normal operation [1].

\section{Why only Zigbee WSN?}

Zigbee technology is a bidirectional wireless communication technology of short distance, low complexity, low cost, low power consumption, and low data rate, mainly used in automatic control. It mainly works on $2.4 \mathrm{GHz}$ ISM band with $20 \sim 250 \mathrm{kbit} / \mathrm{s}$ data rate, $100 \mathrm{~m} \sim 1.5 \mathrm{~km}$ maximum transmission range, and a typical 100m distance [4]. The technical features include:

(1) Security: Zigbee provides data integrity check and authentication, and uses AES-128 security algorithm. Each application has the flexibility to determine its safety properties.

(2) Reliability: It uses collision avoidance mechanism, and at the same time it reserves a dedicated time slot to require a fixed bandwidth of the communication service, avoid the competition and conflicts when data is sent. MAC layer uses a full confirmation of data transfer mechanisms, and each packet of data sent must wait to receive confirmation.

(3) Low cost: the initial cost of module estimates about US $\$ 6$, and soon will fall between US $\$ 1.5$ and US $\$ 2.5$, and Zigbee Protocol is free of royalties. (4) Power saving: as the duty cycle is very short, transmitting and receiving information has lower power consumption, and using the hibernation mode, Zigbee technology ensures that two $\mathrm{N}$ size batteries can support from 6 months to 2 years. Of course, different applications have power different power consumptions.

(5) High network capacity: a Zigbee network can accommodate a maximum of 65536 devices.

(6) Short delays: enhanced communication delays for delay-sensitive applications. Communication delay and sleep wake up time delay are very short. Typical device search delay is 30ms, typical sleep wake up time delay is $15 \mathrm{~ms}$, and active channel access delay is $15 \mathrm{~ms}$.

Main applications of Zigbee are within short range and data transfer rate among the various electronic equipments is not high. The typical transfer data types are periodical data (such as sensor data), intermittent data (such as lighting control), and repetitive low latency data (such as a mouse).

Because Bluetooth, Wi-Fi, and Zigbee all belong to 802.15 protocols, technical characteristics have many similarities. Characteristics comparison is shown in Table 1. Since transmission distance of Bluetooth and Wi-Fi is less than $100 \mathrm{~m}$, it is a huge obstacle in the larger network. Thus it is difficult to form wireless communication network, while the maximum transmission range of Zigbee is $100 \mathrm{~m} \sim 1.5 \mathrm{~km}$, which is ideal for the establishment of the network. Secondly, it can only accommodate up to 8 nodes in a Bluetooth network, but a typical network needs more devices nodes, and Zigbee can accommodate 65536 nodes.

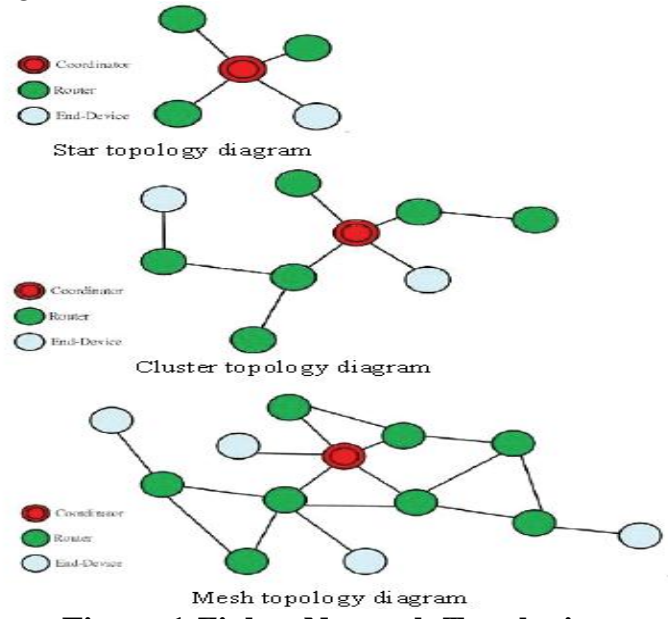

Figure 1 Zigbee Network Topologies 
Finally, module costs of Bluetooth and Wi-Fi are relatively high. In addition, the power consumption of Bluetooth and Wi-Fi compared to Zigbee is much higher, because Zigbee is low cost and very low power consumption. Although transmission data rate of Bluetooth and Wi-Fi is higher than that of Zigbee. But the $250 \mathrm{kbit} / \mathrm{s}$ data rate of Zigbee is enough for use in automatic meter reading network, thus the Zigbee technology is selected.

Table 1 Comparison of Zigbee, Bluetooth, and Wi-Fi

\begin{tabular}{|l|l|l|l|}
\hline \multicolumn{1}{|c|}{ Performance } & \multicolumn{1}{|c|}{ ZigBee } & \multicolumn{1}{c|}{ Bluetooth } & \multicolumn{1}{c|}{ Wi-Fi } \\
\hline $\begin{array}{l}\text { Working } \\
\text { frequency }\end{array}$ & $\begin{array}{l}2.4 \mathrm{GHz} \\
868 / 915 \mathrm{MHz}\end{array}$ & $2.4 \mathrm{GHz}$ & $2.4 \mathrm{GHz}$ \\
\hline System resource & $4 \mathrm{Kbyte} 32 \mathrm{Kbyte}$ & $250 \mathrm{Kbyte}$ & $1 \mathrm{Mbyte}$ \\
\hline Comm range & $0.1 \sim 1.5 \mathrm{~km}$ & $0.1 \mathrm{~km}$ & $0.1 \mathrm{~km}$ \\
\hline Data rate & $250 \mathrm{Kbps}$ & $1 \mathrm{Mbps}$ & $11 \mathrm{Mbps}$ \\
\hline $\begin{array}{l}\text { Max. network } \\
\text { nodes }\end{array}$ & 65536 & 8 & 32 \\
\hline Wake-up time & $30 \mathrm{~ms}$ & $10 \mathrm{~s}$ & $3 \mathrm{~s}$ \\
\hline Encryption & 128 bits AES & 128 bits & SSID \\
\hline $\begin{array}{l}\text { Low power } \\
\text { consumption }\end{array}$ & Support & No support & No support \\
\hline
\end{tabular}

\section{How Useful is UML in Real Time Embedded Design?}

The traditional development process for embedded systems is usually cyclic. The engineer often prototypes an algorithm, tests it on a specific hardware architecture, and then refines the software to make most efficient use of the underlying hardware. If software is to be embedded, this type of development can be very expensive and time consuming as performance analysis is done after system components have been functionally tested and integrated. These complexities of the embedded systems are increasing with the demand of more functionality to be introduced in the systems with limited resources and short time-to-market concepts at lower costs. These issues in turn can be handled by using the reusable components in the systems which can increase the productivity and as well as the reliability. Already the model driven and component based approaches are being used in the development of real-time embedded systems as compared to the traditional sequential approaches. A system is represented in UML using multiple models through the 13diagrams types. Each model describes the system from a distinctly different perspective. At the top level the following three kinds of views of a system are defined in UML:

- Structural Classification: In this view, the various objects or components and their relationships with each other are considered. It represents the static view of the system through class diagrams, use case view through use case diagrams, the implementation view through component and deployment diagrams.

- Dynamic Behaviour: It describes mainly the application's dynamic behaviour i.e. the behaviour over time. This view includes the state machine view through state machine diagrams, the activity view through activity diagrams and the interaction view through sequence or collaboration diagrams.

- Model Management: This view describes the organization of the system models into hierarchical units. It uses the class diagrams to represent the organization. The model management view uses the package construct to cross the other views and organises the application's models during development and configuration control.

Two types of views of the system i.e. static and behavioural one have also been described [3]. The static view of the system represents the entities in a system and the relationships among them. The class diagrams, object diagrams, deployment diagrams and component diagrams are used to construct the static model of a system. Behavioural views show how a system behaves over time by showing the order of happening of things, the conditions under which these happen and the interactions between them. The state charts, use cases, sequence diagrams, collaboration diagrams and activity diagrams are used to construct the behavioural model of the system. Because of the increasing complexity of the systems, there are two main risks dominating in the real-time systems design. The first one is the risk of functionality that is caused by incompletion or misunderstanding the requirements from the user. The second one is the risk of performance. It may arise from insufficient performance evaluation in the early stages of the design itself. As a consequence, it does not fulfill our expectations concerning the needed functionality and performance. These both things if uncovered or realized after the development of the system may cause heavy costs to come back to the design of the system with required specifications. 
The wireless automatic reservoir monitoring and controlling system described in this article can send data from a number of Sensor nodes to the collector through RS-485 communication protocol, and transfer the data to Zigbee /GPRS Gateways through Zigbee Wireless communication network, then back to PC or the database in RS-232 communication format via GPRS as shown in Fig. 2. The wireless communication network in the middle uses Zigbee modules in combination with GPRS remote communication devices developed by ICP DAS, forming a wireless communication network. The Detailed hierarchical order and communication pattern among the blocks is shown in Fig.3. It is a typical distributed control system, and it has a tree network structure. The system can be divided into three layers: the first is the upper communication network between system management center and the concentrator; the second layer is the middle communication network between the concentrator and the collector; the third layer is the bottom communication network between the collector with the meter and other instruments. The upper communication network can use various types of communication networks such as 3G, GRPS, VPN special network, telephone network and other communication, which are wide-area communications network technologies; the middle communication network can use existing wiring systems, such as: telephone network, cable television, power network and wireless communications networks, and it can also use various network resources, such as residential cabling system (PDS). The bottom communication can use PLC transmission mode, 485 mode and wireless mode.

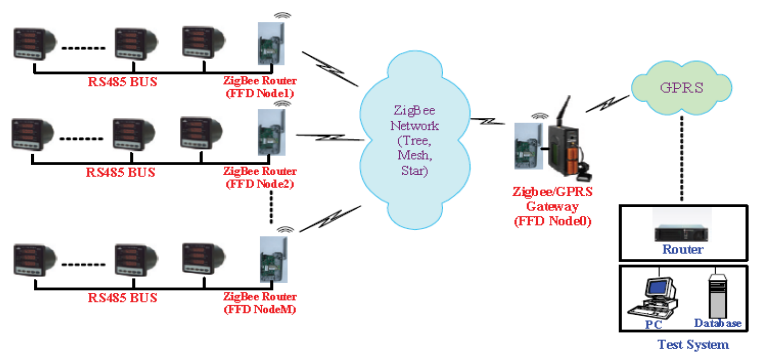

Figure 2 System structure of wireless automatic meter reading system

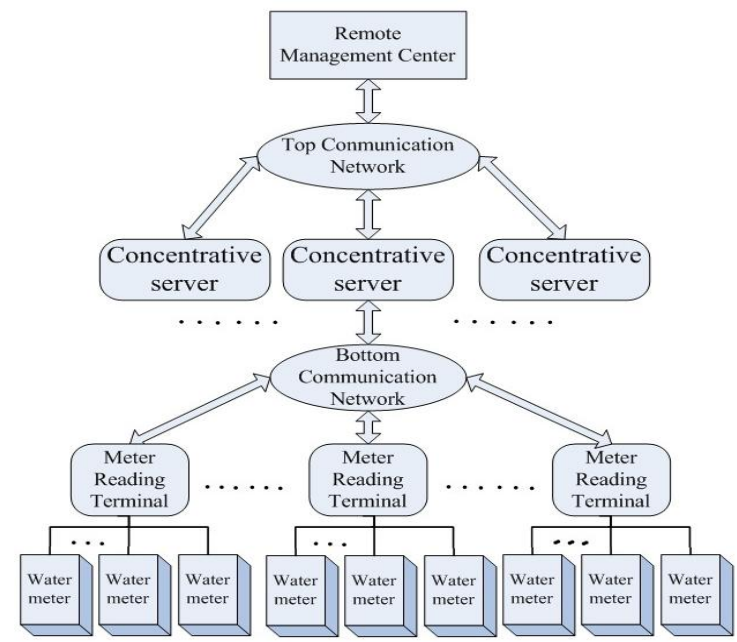

Figure 3. Detailed Hierarchy of the Blocks

\section{Software Designing}

Zigbee end device reads measurement information in multifunction sensor or water meter by UART, and transmits them to the network coordinator via Zigbee wireless network, thus it can realize the wireless sensor reading of the network coordinator. 


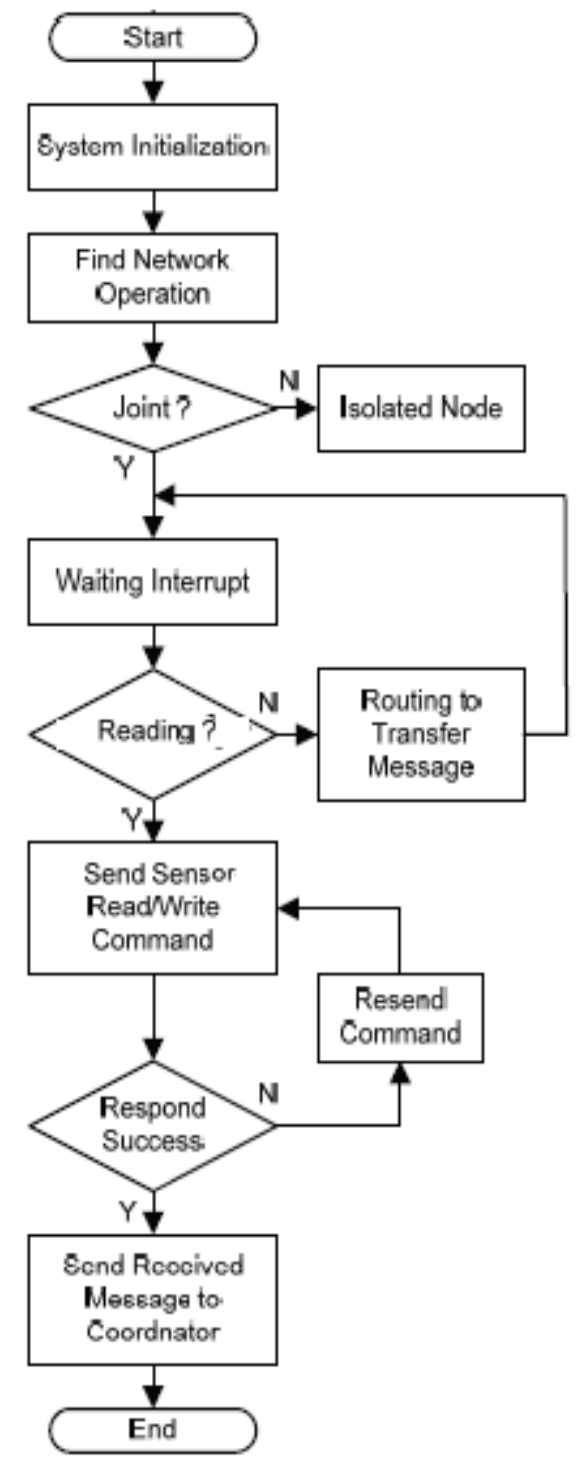

Figure 4 Program flowchart of Zigbee end device (Wireless Sensor Node)

Meanwhile, the network coordinator can transfer clock-correcting command to sensor node through Zigbee end device to calibrate the parameters. Therefore, the end device must be able to communicate with the Wireless sensor node in order to read data and the coordinator in order to send data. The coordinator also has to know which end node that sends the data so the data can be read accurately. Each of Zigbee modules has a unique 64-bit permanent address, so the addressing won't be much difficult. Fig. 4 shows the program flowchart of end device.

\section{UML Modeling of the Embedded Design}

When this Wireless Reservoir monitoring system is modeled on UML Several Issues in the embedded designs were answered. The first phase in development of an embedded system either hardware or software is functional requirements capturing. This basically defines what the system is supposed to do. In case of UML, the requirements are captured using use-case diagrams from external actors' point of view. A use-case tells what the system does for each actor. In the present system (Stabilization System), the actor can be a human user as well as the external I/O devices. The use-case diagram for stabilization system is shown in Figure 5. The usecases shown in the frame stab_control_system are the functions identified that the system is supposed to do. 


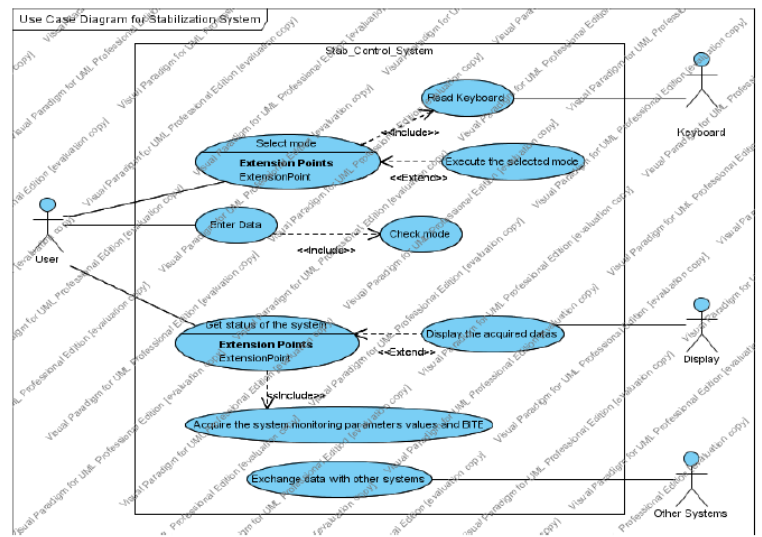

Figure 5. Use Case diagram for stabilization system

The real-time embedded systems continuously interact with the environment and respond to the events. Therefore, it is very much essential to understand the interface between the system and the external environment. The static modelling is done for showing these interfaces and to describe the static structure of the system by developing a system context diagram. A system context diagram provides a more detailed view of the system boundary than a use-case especially for real-time systems. The system context class diagram for stab_control_system is shown in Figure 6. The use case diagrams of the three parts of our design Sensor, Data Controller \& Main controller are shown in Fig.7.

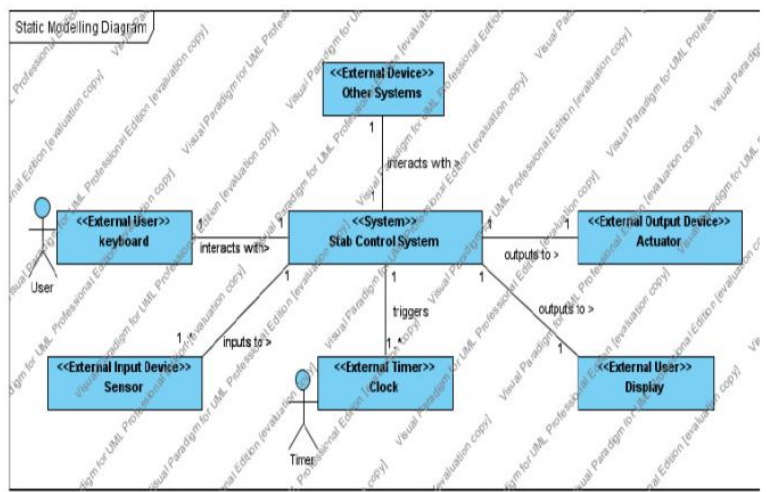

Figure 6 Class Diagram for Stab_Control_System
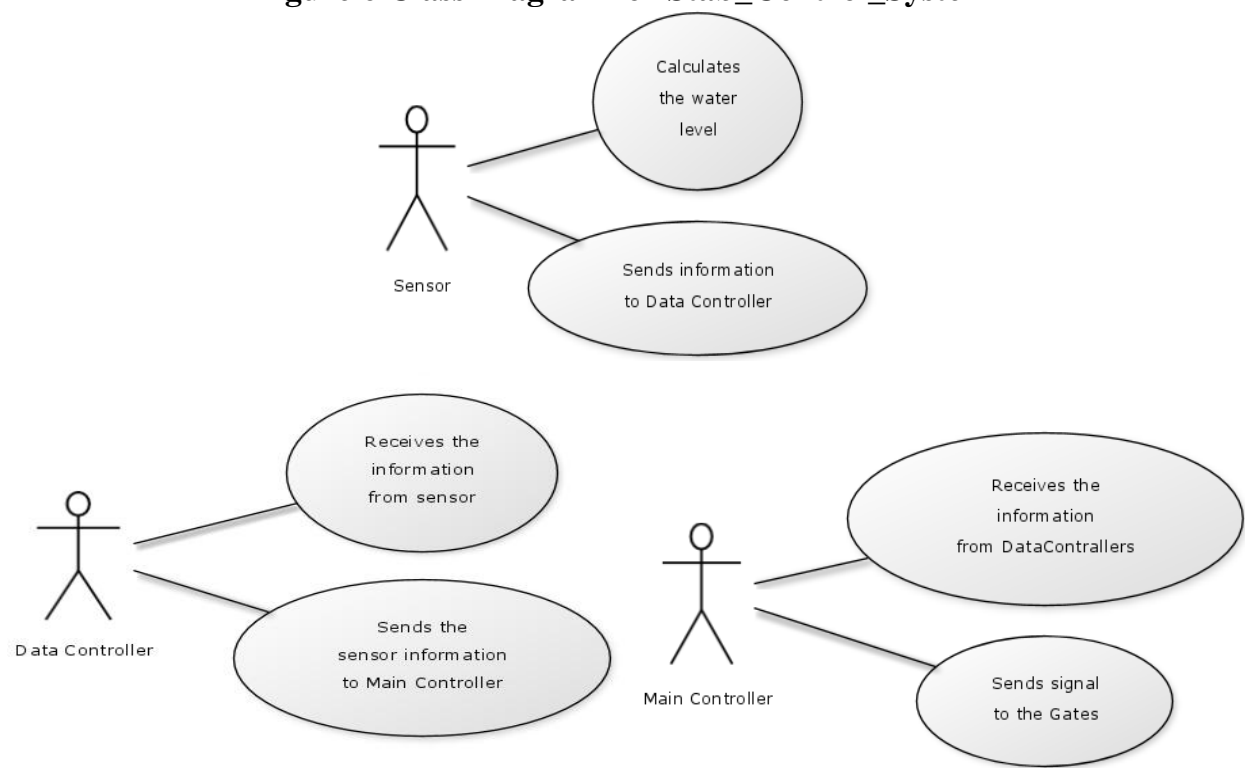

Figure 7 Use case diagrams of the models 


\section{Conclusions}

We have presented the requirements and analysis modeling of a Reservoir monitoring control system using object oriented concept using UML/COMET method. Through these implementations, it has been found that the UML standard is very useful in requirements capturing, decomposing the system into objects and defining their relationships. The control system application models have been developed using three UML modeling mechanisms: use case modeling, object modeling, and dynamic modeling respectively. Through requirements modeling and analysis modeling, the usability of the Use Case, Class/object, and Collaboration and State-Chart diagrams has been exploited. The models presented in this work are obtained after lot of iteration going forward and backward between static and dynamic models. The models have been developed irrespective of the target platforms on which finally they will be executed, hence, enabling their reuse over different operating platforms. The approach used in this work shows the possible adoption of UML in the embedded system design.

The successful development of the wireless reservoir monitoring system described in this article is based on the high performance, extremely low power consumption, high level of integration, and low price of Zigbee technology. The technology has strong market competitiveness. Zigbee wireless system uses short-range wireless communication and computer network technologies to read and process metering data automatically. It can not only save human resources, but also improve the accuracy and instantaneity of the monitoring system. It enables management sector to timely and accurately access power consumption.

\section{Acknowledgements}

The authors would like to thank the anonymous reviewers for their comments which were very helpful in improving the quality and presentation of this paper.

\section{References}

[1] G. Martin, L. Lavagno, and J. Louis-Guerin, "Embedded UML: a merger of real-time UML and codesign", CODES 2001, Copenhagen, April 2001, pp.23-28

[2] Rumbaugh, J., Jacobson, I. and Booch, G. 1999. The Unified Modeling Language - Reference Manual. Reading, MA: Addison Wesley Longman.

[3] Holt. J. 2001. UML for Systems Engineering. United Kingdom: The Institution of Electrical Engineers.

[4] Hofmann, P. P. and Schurr, A., 2002. OMER - Object-oriented Modeling of Embedded Real-Time

[5] Systems. Post-proceedings of OMER-1: May 28/29 1999, Herrsching am Ammersee and OMER-2: May 10-12 2001, Herrsching am Ammersee.

[6] H. Gomma, Designing Concurrent, Distributed, and Real-Time Application with UML, Addison-Wesley, 2000

[7] Safaric S., Malaric K., ZigBee Wireless Standard, Proc. of the 48th International Symposium ELMAR-2006, Zadar Croatia, 1 (2006), 259-262

[8] Primicanta, A.H., Nayan, M.Y., Awan, M., ZigBee-GSM based Automatic Meter Reading system, 2010 International Conference on Intelligent and Advanced Systems (ICIAS), Kuala Lumpur, Malaysia, 1 (2010), 1-5

[9] Tatsiopoulos, C., Ktena, A., A Smart ZIGBEE Based Wireless Sensor Meter System, 16th International Conference on Systems, Signals and Image Processing (IWSSIP), Chalkida, Greece, 1 (2009), 1-4

[10] Lee J.D., Nam K.Y., Jeong S.H., Choi S.B., Ryoo H.S., Kim D.K., Development of ZigBee Based Street Light Control System, Proc. of the Power System Conference and Exposition, Atlanta GA, 3 (2006), 2236-2240 